University of Wollongong

Research Online

Faculty of Engineering - Papers (Archive)

Faculty of Engineering and Information

Sciences

$1-1-2010$

\title{
A polynomial chaos-based kalman filter approach for parameter estimation of mechanical systems
}

\author{
Emmanuel D. Blanchard \\ Virginia Polytechnic Institute and State University, Blacksburg, Virginia, USA, eblancha@uow.edu.au \\ Corina Sandu \\ Virginia Tech Department of Mechanical Engineering \\ Adrian Sandu \\ Virginia Tech Department of Computer Science
}

Follow this and additional works at: https://ro.uow.edu.au/engpapers

Part of the Engineering Commons

https://ro.uow.edu.au/engpapers/572

\section{Recommended Citation}

Blanchard, Emmanuel D.; Sandu, Corina; and Sandu, Adrian: A polynomial chaos-based kalman filter approach for parameter estimation of mechanical systems 2010.

https://ro.uow.edu.au/engpapers/572

Research Online is the open access institutional repository for the University of Wollongong. For further information contact the UOW Library: research-pubs@uow.edu.au 


\section{DETC2007-34600}

\section{A POLYNOMIAL-CHAOS-BASED BAYESIAN APPROACH FOR ESTIMATING UNCERTAIN PARAMETERS OF MECHANICAL SYSTEMS}

\author{
Emmanuel Blanchard \\ Advanced Vehicle Dynamics Lab. \\ Center for Vehicle Systems and Safety \\ Virginia Polytechnic Institute and State \\ University, Blacksburg, VA 24061 \\ eblancha@vt.edu
}

\author{
Corina Sandu \\ Advanced Vehicle Dynamics Lab. \\ Center for Vehicle Systems and Safety \\ Virginia Polytechnic Institute and State \\ University, Blacksburg, VA 24061 \\ csandu@vt.edu
}

\author{
Adrian Sandu \\ Computer Science Department \\ Virginia Polytechnic Institute and State \\ University, Blacksburg, VA 24061 \\ sandu@cs.vt.edu
}

Keywords: Parameter Estimation, Polynomial Chaos, Collocation, Bayesian Estimation, Hammersley, Halton, Vehicle Dynamics.

\section{ABSTRACT}

In this study, a new computational approach for parameter identification is proposed based on the application of the polynomial chaos theory. The polynomial chaos method has been shown to be considerably more efficient than Monte Carlo in the simulation of systems with a small number of uncertain parameters. In the new approach presented in this paper, the maximum likelihood estimates are obtained by minimizing a cost function derived from the Bayesian theorem. Direct stochastic collocation is used as a less computationally expensive alternative to the traditional Galerkin approach to propagate the uncertainties through the system in the polynomial chaos framework. The new parameter estimation method is illustrated on a four degree-of-freedom roll plane model of a vehicle in which the vertical stiffnesses of the tires are estimated from periodic observations of the displacements and velocities across the suspensions. The results obtained with this approach are close to the actual values of the parameters even when only measurements with low sampling rates are available. The accuracy of the estimations has been shown to be sensitive to the number of terms used in the polynomial expressions and to the number of collocation points, and thus it may become computationally expensive when a very high accuracy of the results is desired. However, the noise level in the measurements affects the accuracy of the estimations as well. Therefore, it is usually not necessary to use a large number of terms in the polynomial expressions and a very large number of collocation points since the addition of extra precision eventually affects the results less than the effect of the measurement noise. Possible applications of this theory to the

field of vehicle dynamics simulations include the estimation of mass, inertia properties, as well as other parameters of interest.

\section{INTRODUCTION AND BACKGROUND}

The polynomial chaos theory has been shown to be consistently more efficient than Monte Carlo simulations in order to assess uncertainties in mechanical systems $[9,10]$. This paper extends the polynomial chaos theory to the problem of parameter estimation, and applies it to a four degree of freedom roll plane model of a vehicle. Parameter estimation is an important problem, because many parameters simply cannot be 
measured physically with good precision, especially in real time applications. The method presented in this paper has the advantage of being able to deal with non-Gaussian parametric uncertainties.

Parameter estimation is a very difficult problem, especially for large systems, and a lot of effort devoted to it would be needed. Estimating a large number of parameters often proved to be computationally too expensive. This has led to the development of techniques determining which parameters affect the system's dynamics the most, in order to choose the parameters that are important to estimate [12]. Sohns, et al. [12] proposed the use of activity analysis as an alternative to sensitivity-based and principal component-based techniques. Their approach combines the advantages of the sensitivitybased techniques (i.e., being efficient for large models) and the sensitivity-based techniques (i.e., keeping parameters that can be physically interpreted). Zhang and Lu [13] combined the Karhunen-Loeve decomposition and perturbation methods with polynomial expansions in order to evaluate higher-order moments for saturated flow in randomly heterogeneous porous media.

\section{PROBLEM FORMULATION}

Optimal parameter estimation combines information from three different sources: the physical of evolution (encapsulated in the model), the reality (as captured by the observations), and the current best estimate of the parameters (all with associated errors). Consider a dynamic model which advances the state in time

$$
y^{k}=M\left(t^{k-1}, y^{k-1}, \theta\right), \quad y^{0}=y\left(t^{0}\right), \quad k=1,2, \ldots, N
$$

The state of the model $y^{k}$ at time moment $t^{k}$ depends implicitly on the set of parameters $\theta \in \mathfrak{R}^{p}$, possibly uncertain (the model has $\mathrm{n}$ states and $\mathrm{p}$ parameters). $M$ is the discrete model solution operator which integrates the model equations forward in time (starting from state $y^{k-1}$ at time $t^{k-1}$ to state $y^{k}$ at time $t^{k}$ ).

Using polynomial chaoses the uncertain parameters can be modeled explicitly as functions of a set of random variables $\xi \in \Omega \subset \Re^{p}$ with a joint probability density function $\rho(\xi)$. The explicit dependency is in the form of an expansion in terms of orthogonal polynomial basis functions

$$
\theta(\xi)=\sum_{i=1}^{S} \theta^{i} \phi^{i}(\xi), \quad y^{k}(\xi)=\sum_{i=1}^{S}\left(y^{k}\right)^{i} \phi^{i}(\xi)
$$

The time evolution of the uncertain model state can be obtained from (1) and (2) via a Galerkin or collocation approach $[9,10]$.

For parameter estimation it is convenient to formally extend the model state to include the model parameters and extend the model with equations for parameters (such that parameters do not change during the model evolution)

$$
\left[\begin{array}{c}
y^{k} \\
\theta^{k}
\end{array}\right]=\left[\begin{array}{c}
M\left(t^{k-1}, y^{k-1}, \theta^{k-1}\right) \\
\theta^{k-1}
\end{array}\right]
$$

The optimal estimation of the uncertain parameters is thus reduced to the problem of optimal state estimation. Observations of quantities that depend on system state are available at discrete times $t^{k}$

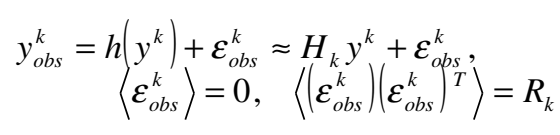

where $y_{o b s}^{k} \in \mathfrak{R}^{m}$ is the observation vector at $t^{k}, h$ is the (model equivalent) observation operator and $H_{k}$ is the linearization of $h$ about the solution $y^{k}$. Note that there are $\mathrm{m}$ observations for the $\mathrm{n}$-dimensional state vector, and that typically $\mathrm{m}<\mathrm{n}$. Each observation is corrupted by observational (measurement and representativeness) errors [2]. We denote by $\langle\cdot\rangle$ the ensemble average over the observation uncertainty $\varepsilon_{o b s}^{k} \in \Re^{m}$ space. The observational error is the experimental uncertainty associated with the measurements and is usually considered to have a Gaussian distribution with zero mean and a known covariance matrix $R_{k}$.

The aim of data assimilation is to find $P\left[y\left(t^{k}\right) \mid y_{o b s}^{k} \ldots y_{o b s}^{0}\right]$, the PDF of the true state at time $t^{k}$ conditioned by all previous observations (including the most recent one).

\section{BAYESIAN APPROACH FOR PARAMETER ESTIMATION}

From Bayes' rule a sequential parameter estimation procedure gives the probability density of the parameter distribution conditioned by all observations as

$$
P\left[y\left(t^{k}\right) \mid y_{o b s}^{k} \ldots y_{o b s}^{0}\right]=\frac{P\left[y_{o b s}^{k} \mid y\left(t^{k}\right)\right] \cdot P\left[y\left(t^{k}\right) \mid y_{o b s}^{k-1} \ldots y_{o b s}^{0}\right]}{\int P\left[y_{o b s}^{k} \mid y\right] \cdot P\left[y \mid y_{o b s}^{k-1} \ldots y_{o b s}^{0}\right] d y}
$$


where $P\left[y\left(t^{k}\right) \mid y_{o b s}^{k-1} \ldots y_{o b s}^{0}\right]$ is the PDF of the latest observational error, $P\left[y\left(t^{k}\right) \mid y_{o b s}^{k} \ldots y_{o b s}^{0}\right]$ is the "model forecast PDF" (conditioned by all previous observations minus the most recent one) and $P\left[y\left(t^{k}\right) \mid y_{o b s}^{k} \ldots y_{o b s}^{0}\right]$ is the "assimilated PDF".

For simplicity denote by $y$ the current state of the system (the best estimation obtained using all previous observations $y_{o b s}^{k-1} \ldots y_{o b s}^{0}$ ) and by $z=y_{o b s}^{k}$ the latest, yet-to-be-used set of observations. Moreover, consider that the observational error has a Gaussian distribution with covariance R. Then Bayes' formula becomes

$$
P[y \mid z]=\frac{P[z \mid y] \cdot P[y]}{\int_{\mathfrak{R}^{n}} P[z \mid w] \cdot P[w] d w}=\frac{e^{-\frac{1}{2}(z-H y)^{T} R^{-1}(z-H y)} \cdot P[y]}{\int_{\mathfrak{R}^{n}} e^{-\frac{1}{2}(z-H w)^{T} R^{-1}(z-H w)} \cdot P[w] d w}
$$

The unconditional probability density $P[y]$ is the PDF of the current system state, and is implicitly represented by the polynomial chaos expansion of the state $y=y(\xi)$. Moreover, integration against this probability density can be evaluated by integration in the independent random variables

$$
\int_{\mathfrak{R}^{n}} f(y) \cdot P[y] \cdot d y=\int_{\Omega} f(y(\xi)) \cdot \rho(\xi) \cdot d \xi \text { for any } f(\cdot)
$$

The denominator can be evaluated by a multidimensional integration. However, in our approach, there is no need to evaluate this scaling factor, since its omission does not change the estimation procedure. (The omission of this scaling factor is equivalent to adding a constant to the function we minimize, and this does not change the result of the minimization procedure).

The mean of the best state estimate that uses the new observations $\mathrm{z}$ is obtained from Bayes formula as

$$
\begin{aligned}
\langle y\rangle & =\int_{\mathfrak{R}^{n}} y \cdot P[y \mid z] \cdot d y \\
& =\frac{1}{\operatorname{den}} \int_{\mathfrak{R}^{n}} y \cdot e^{-\frac{1}{2}(z-H y)^{T} R^{-1}(z-H y)} \cdot P[y] \cdot d y \\
& =\frac{1}{\operatorname{den}} \int_{\Omega} y(\xi) \cdot e^{-\frac{1}{2}(z-H y(\xi))^{T} R^{-1}(z-H y(\xi))} \cdot \rho(\xi) \cdot d \xi
\end{aligned}
$$

The multidimensional integration formula can be applied to evaluate this integral as well. Similarly, the variance of this estimate as well as its higher moments can be derived from Bayes' formula with the help of multidimensional integration. For the parameter estimation the Bayes' formula specializes to:

$$
P[\theta \mid z]=\frac{P[z \mid \theta] \cdot P[\theta]}{\operatorname{den}}=\frac{e^{-\frac{1}{2}(z-H y(\theta))^{T} R^{-1}(z-H y(\theta))} \cdot P[\theta]}{\int_{\mathfrak{R}^{m}} e^{-\frac{1}{2}(z-H w)^{T} R^{-1}(z-H w)} \cdot P[\theta] d \theta}
$$

Note that the aposteriori probability defined by Bayes formula can be written (in principle) as a function of the independent random variables $\xi$

$$
\hat{\rho}(\xi)=P[\xi \mid z]=\frac{e^{-\frac{1}{2}(z-H y(\xi))^{T} R^{-1}(z-H y(\xi))} \cdot \rho(\xi)}{\int_{\Omega} e^{-\frac{1}{2}(z-H w(\xi))^{T} R^{-1}(z-H w(\xi))} \cdot \rho(\xi) d \xi}
$$

In this setting polynomial chaos is used to model the $a$ priori pdf of the parameters; the Bayes formula is employed to obtain the a posteriori pdf (i.e., the pdf conditioned by the observations).

The maximum likelihood estimate is given by that realization of the parameters (that value of $\xi$ ) which maximizes, or, equivalently, minimizes $-\log (P[\theta \mid z])$ :

$$
\min _{\xi \in \Omega} J=\frac{1}{2}(z-H y(\xi))^{T} R^{-1}(z-H y(\xi))-\log (\rho(\xi))
$$

Note that for $\xi \notin \Omega$ we have $\rho(\xi)=0$ and cost the function $\mathrm{J}$ becomes infinite.

\section{APPLICATION TO A MECHANICAL SYSTEM}

\section{Roll Plane Modeling of a Vehicle}

The model used to apply the theory presented in this article is based on the four degree of freedom roll plane model of a vehicle used in [11], which is shown in Figure 1. The difference is that the suspension dampers and the suspension springs used in this study are nonlinear and the tire vertical stiffnesses $k_{t 1}$ and $k_{t 2}$ are assumed to be uncertain.

If $x$ is the relative displacement across the suspension spring with a stiffness $k_{i}(i=1,2)$, the force across the suspension spring is given by:

$$
F_{K_{i}}(x)=k_{i} x+k_{i, 3} x^{3}, \quad i=1,2
$$

If $v$ is the relative velocity across the damper with a damping coefficient $c_{i}(i=1,2)$, the force across the damper is given by:

$$
F_{C_{i}}(v)=c_{i}(0.2 \tanh (10 v))
$$

It is assumed that the probability density functions of the values of $k_{t 1}$ and $k_{t 2}$ can be represented with a $\operatorname{Beta}(1,1)$ distribution, with an uncertainty of $+/-20 \%$. 


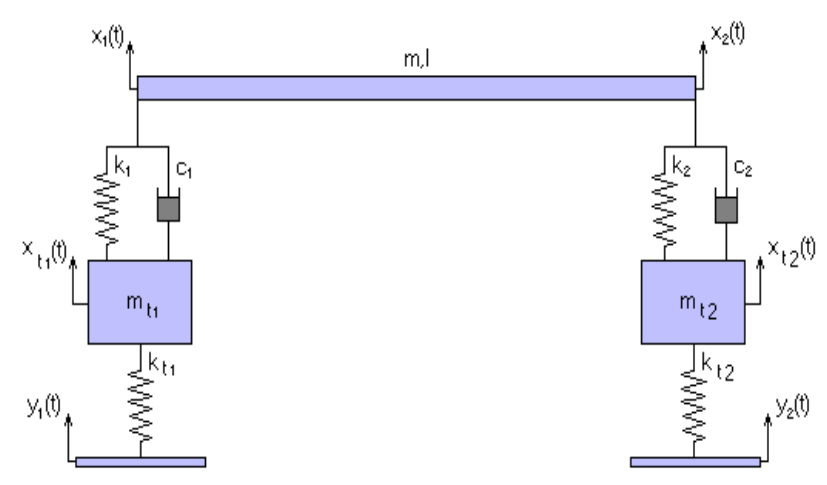

Figure 1. Four Degree of Freedom Roll Plane Model [11].

The body of the vehicle is represented as a bar of mass $m$ (sprung mass) and length $l$ that has a moment of inertia $I$. The unsprung masses, i.e., the mass of each tire/axle combination, are represented by $m_{t 1}$ and $m_{t 2}$.

The motion variables $x_{1}$ and $x_{2}$ correspond to the vertical position of each side of the vehicle body, while the motion variables $x_{t 1}$ and $x_{t 2}$ correspond to the position of the tires.

The inputs to this system are $y_{1}$ and $y_{2}$, which represent the road profile under each wheel.

The equations of motion of the system are

$$
\begin{gathered}
\begin{array}{r}
\frac{m}{2}\left(\ddot{x}_{2}+\ddot{x}_{1}\right)+F_{K_{1}}\left(x_{1}-x_{t 1}\right)+F_{K_{2}}\left(x_{2}-x_{t 2}\right) \\
\quad=F_{C_{1}}\left(\dot{x}_{t 1}-\dot{x}_{1}\right)+F_{C_{2}}\left(\dot{x}_{t 2}-\dot{x}_{2}\right)
\end{array} \\
\begin{array}{r}
\frac{I}{l}\left(\ddot{x}_{2}-\ddot{x}_{1}\right)+\frac{l}{2} F_{K_{2}}\left(x_{2}-x_{t 2}\right)-\frac{l}{2} F_{K_{1}}\left(x_{1}-x_{t 1}\right) \\
=\frac{l}{2} F_{C_{1}}\left(\dot{x}_{1}-\dot{x}_{t 1}\right)-\frac{l}{2} F_{C_{2}}\left(\dot{x}_{2}-\dot{x}_{t 2}\right)
\end{array} \\
m_{t 1} \ddot{x}_{t 1}+F_{K_{1}}\left(x_{t 1}-x_{1}\right)+F_{C_{1}}\left(\dot{x}_{t 1}-\dot{x}_{1}\right)=k_{t 1}\left(y_{1}-x_{t 1}\right) \\
m_{t 2} \ddot{x}_{t 2}+F_{K 2}\left(x_{t 2}-x_{2}\right)+F_{C_{2}}\left(\dot{x}_{t 2}-\dot{x}_{2}\right)=k_{t 2}\left(y_{2}-x_{t 2}\right)
\end{gathered}
$$

where $F_{K_{1}}, F_{K_{2}}, F_{C_{1}}$, and $F_{C_{2}}$ are defined in Equations (12) and (13).

The parameters used in this study are shown in Table 1. They are the parameters used by [11], with the addition of nonlinearities and uncertainties.

Table 1. Vehicle Parameters

\begin{tabular}{|c|c|c|}
\hline Parameter & Description & Value \\
\hline$m$ & Mass of the Roll Bar & $580 \mathrm{~kg}$ \\
\hline$m_{t 1}, m_{t 2}$ & Mass of the tire/axle & $36.26 \mathrm{~kg}$ \\
\hline$c_{1}, c_{2}$ & Damping coefficients & $710.70 \mathrm{~N} \mathrm{~s} / \mathrm{m}$ \\
\hline
\end{tabular}

\begin{tabular}{|c|c|c|}
$k_{1}, k_{2}$ & $\begin{array}{c}\text { Spring constants - } \\
\text { linear component }\end{array}$ & $19,357.2 \mathrm{~N} / \mathrm{m}$ \\
\hline$k_{1,3}, k_{2,3}$ & $\begin{array}{c}\text { Spring constants - } \\
\text { cubic component }\end{array}$ & $15,000 \mathrm{~N} / \mathrm{m}^{3}$ \\
\hline$l$ & $\begin{array}{c}\text { Length of the Roll } \\
\text { Bar }\end{array}$ & $1.524 \mathrm{~m}$ \\
\hline$I$ & Inertia of the Roll Bar & $63.3316 \mathrm{~kg} \mathrm{~m}{ }^{2}$ \\
\hline$k_{t 1}, k_{t 2}$ & $\begin{array}{c}\text { Tires vertical } \\
\text { stiffnesses }\end{array}$ & $\begin{array}{c}96,319.76 \mathrm{~N} / \mathrm{m} \\
+/-20 \%, \text { with } \\
\text { Beta (1, 1) distribution }\end{array}$ \\
\hline
\end{tabular}

The uncertainties of $20 \%$ on the values of $k_{t 1}$ and $k_{t 2}$ can be represented as:

$$
\begin{aligned}
& k_{t 1}=k_{t 1 \text { nom }}\left(1+0.20 \xi_{1}\right), \quad \xi_{1} \in[-1,1] \\
& k_{t 2}=k_{t 2 \text { nom }}\left(1+0.20 \xi_{2}\right), \quad \xi_{2} \in[-1,1]
\end{aligned}
$$

where $k_{t 1 \text { nom }}$ and $k_{t 2 n o m}$ are the nominal values of the vertical stiffnesses of the tires $\left(k_{t 1 \text { nom }}=96,319.76 \mathrm{~N} / \mathrm{m}\right.$ and $k_{t 2 \text { nom }}=96,319.76 \mathrm{~N} / \mathrm{m}$ ).

The distributions of the uncertainties related to the values of $k_{t 1}$ and $k_{t 2}$, defined on the interval $[-1,1]$, are represented in Figure 2. They have the following Probability Density Functions (PDFs):

$$
w\left(\xi_{i}\right)=\frac{3}{4}\left(1-\xi_{i}^{2}\right), \quad i=1,2
$$

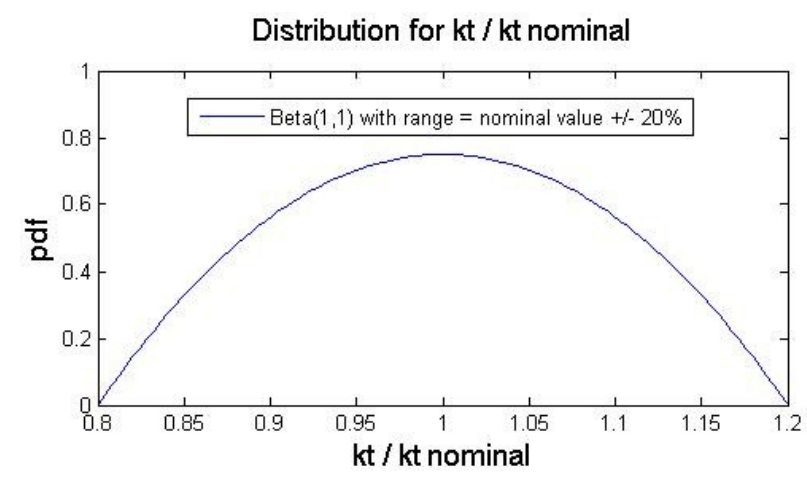

Figure 2. Beta $(1,1)$ Distribution

\section{Collocation Points}

The generalized polynomial chaos theory is explained in [8], in which direct stochastic collocation is proposed as a less expensive alternative to the traditional Galerkin approach. The collocation approach consists of imposing that the equation system holds at a given set of collocation points. If the 
polynomial chaos expansions contain 15 terms for instance, then at least 15 collocation points are needed in order to have at least 15 equations for 15 unknown polynomial chaos coefficients. It is desirable to have more collocation points than polynomial coefficients to solve for. In that case a least-squares algorithm is used to solve the system with more equations than unknowns.

Unless otherwise specified, the polynomial chaos expansions of $k_{t 1}$ and $k_{t 2}$ will use 15 terms. All the other variables affected by the uncertainties on $k_{t 1}$ and $k_{t 2}$ will be modeled by a polynomial chaos expansion using 15 terms as well. The collocation approach is the one used in this study. It requires at least 15 collocation points to derive the coefficients associated to each of the 15 terms of the different polynomial chaos expansions.

Unless otherwise specified, 30 collocation points will be used to derive the coefficients associated to each of the 15 terms of the different polynomial chaos expansions. The collocation points used in this study are obtained using an algorithm based on the Halton algorithm [6], which is similar to the Hammersley algorithm [7]. These collocation points for a uniform distribution are shown in Figure 3.

One of the advantages of the Hammersley/Halton points used in this study is that when the number of points is increased, the new set of points still contains all the old points. We therefore know that more points should result in a better approximation. The collocation points for a $\operatorname{Beta}(1,1)$ distribution, which is used in this study, are shown in Figure 4.

The transformation from the collocation points for a uniform distribution to the points for a Beta $(1,1)$ distribution is achieved by applying the inverse Cumulative Distribution Function of the Beta $(1,1)$ distribution. Let's note that there is no collocation point at the boundary, i.e., no point associated with an uncertainty equal to -1 or 1 , which is needed in order to avoid having a cost function equal to infinity.

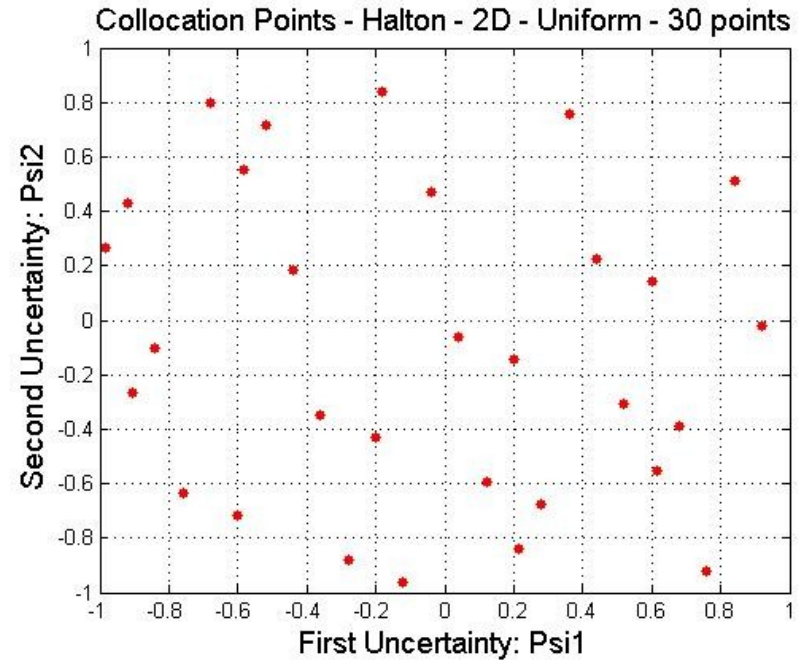

Figure 3. Halton Collocation Points for Uniform Distribution (2-Dimensions, 30 Points)

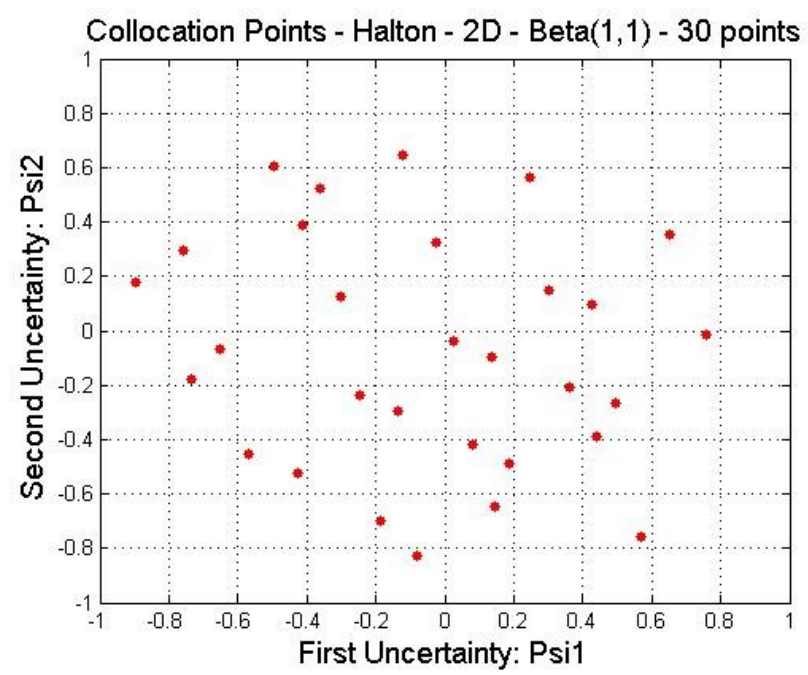

Figure 4. Halton Collocation Points for Beta $(1,1)$ Distribution (2-Dimensions, 30 Points)

\section{Experimental Setting}

In order to assess the efficiency of the polynomial chaos theory for parameter estimation, $k_{t 1}$ and $k_{t 2}$ will be estimated using a plot of four motion variables obtained for a given road input: the displacements across the suspensions $\left(x_{1}-x_{t 1}\right.$ and $\left.x_{2}-x_{t 2}\right)$, and their corresponding velocities $\left(\dot{x}_{1}-\dot{x}_{t 1}\right.$ and $\dot{x}_{2}-\dot{x}_{t 2}$ ). The road profile is shown in Figure 5, and the road input is obtained assuming the vehicle has a constant speed of $16 \mathrm{~km} / \mathrm{h}$ (10 mph). The road profile can be seen as a long speed bump. The first tire is subjected to a ramp at $\mathrm{t}=0$, and reaches a height of $15 \mathrm{~cm}$ (6") for a horizontal displacement of $1 \mathrm{~m}$, then stays at the same height for $1 \mathrm{~m}$, and goes back down to its initial height. The second tire is subjected to the same kind of input, but with a time delay of $20 \%$ and it reaches a maximum height of only $12 \mathrm{~cm}$. 


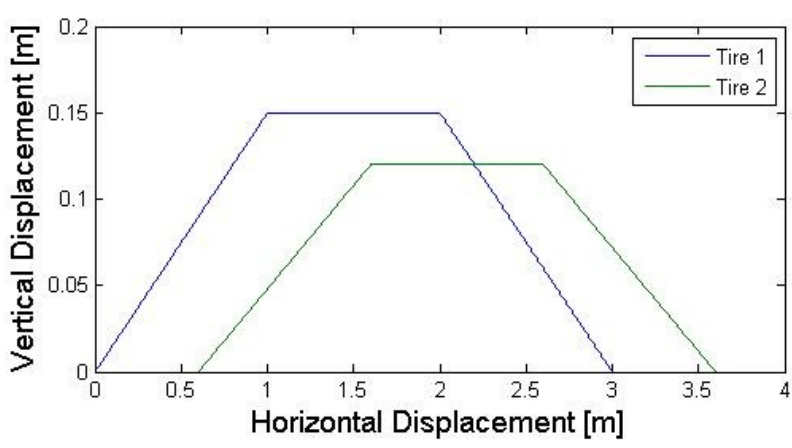

Figure 5. Road Profile

The four motion variables are plotted from $t=0$ to $t=3$ seconds using $k_{t 1 \text { ref }}=100,800 \mathrm{~N} / \mathrm{m}$ and $k_{t 2 \text { ref }}=88,855 \mathrm{~N} / \mathrm{m}$ (i.e., $\xi_{1 \text { ref }}=0.2326$ and $\xi_{2 \text { ref }}=-0.3875$ ) and assuming these values can only be measured with a sampling rate of $0.3 \mathrm{~s}$. However, for the proof of concept of the parameter estimation method presented in this paper, we pretend we do not know the values of $k_{t 1}$ and $k_{t 2}$, the objective being to estimate those values based on the plot of the four motion variables shown in Figure 6. Let's note that 3 seconds of data correspond to a horizontal displacement of 13.33 meters. The end of the speed bump occurs at $\mathrm{t}=0.675 \mathrm{~s}$.
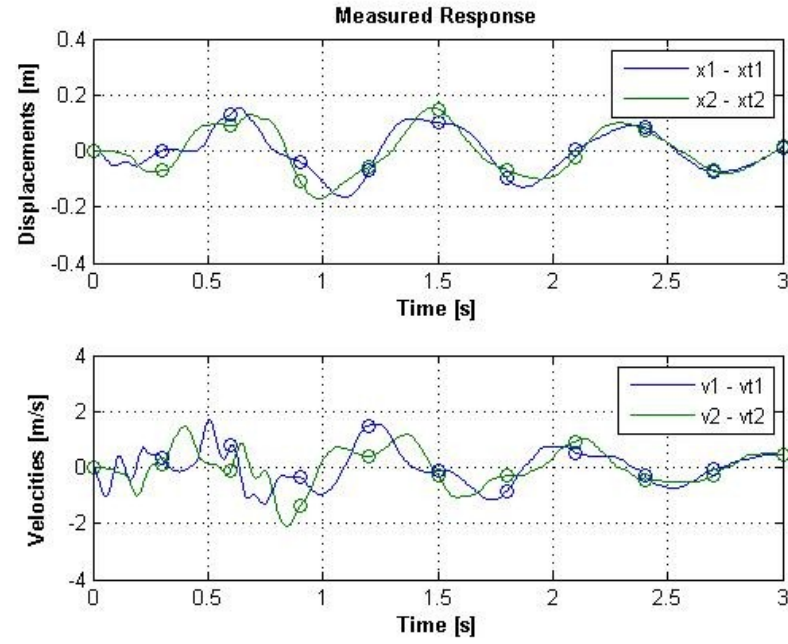

Figure 6. Observed States - Displacements and Velocities

Parameters estimation is performed using the Bayesian approach. In order to work with a realistic set of measurements, a Gaussian measurement noise with zero mean and $1 \%$ variance is added to the observations (for the relative displacements and velocities) shown in Figure 6 before performing parameter estimation.

\section{Results using the Bayesian Approach}

The value of the cost function can be visualized, as shown in Figure 7. A simple Matlab code can estimate the values of $\xi_{1}$ and $\xi_{2}$ (and thus the values of $k_{t 1}$ and $k_{t 2}$ ) corresponding to the minimum value of the cost function.

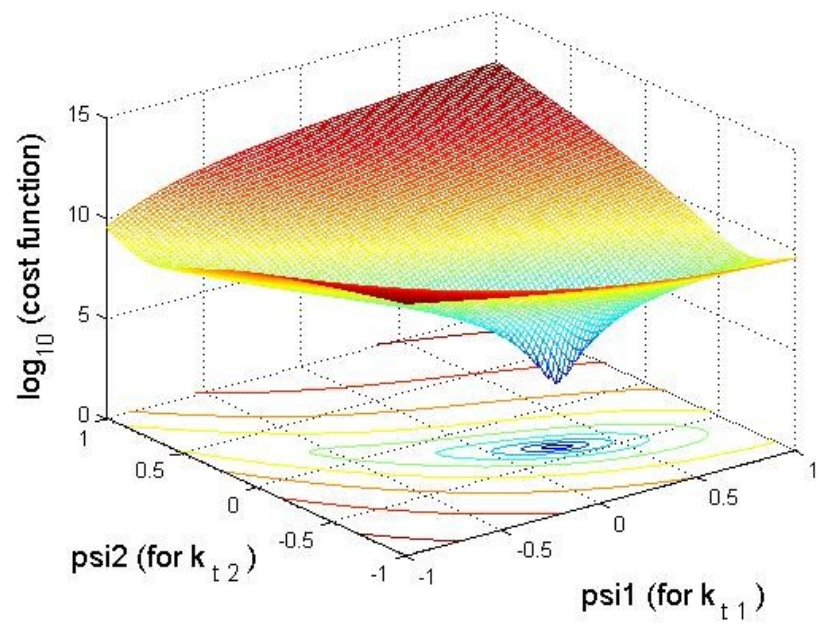

Figure 7. Cost Function Using the Bayesian Approach

The estimated values of $\xi_{1}$ and $\xi_{2}$ obtained using the Bayesian approach are $\xi_{1 \text { est }}=0.2460$ and $\xi_{2 \text { est }}=-0.3783$, i.e., $k_{t 1 \text { est }}=101,059 \mathrm{~N} / \mathrm{m}$ and $k_{t 2 \text { ref }}=89,032 \mathrm{~N} / \mathrm{m}$. The actual values were $\xi_{1 \text { ref }}=0.2326$ and $\xi_{2 \text { ref }}=-0.3875$, i.e., $k_{t 1 \text { ref }}=100,800 \mathrm{~N} / \mathrm{m}$ and $k_{t 2 \text { ref }}=88,855 \mathrm{~N} / \mathrm{m}$. It seems to be a good estimation considering that only 10 measurement points were used and that there is noise associated to the measurements. With a Gaussian measurement noise with zero mean and $0.01 \%$ variance the results would be $\xi_{1 \text { est }}=0.2394$ and $\quad \xi_{2 \text { est }}=-0.3730$, i.e., $\quad k_{t 1 \text { est }}=100,932 \mathrm{~N} / \mathrm{m} \quad$ and $k_{t 2 \text { est }}=89,134 \mathrm{~N} / \mathrm{m}$. It shows that the effects of a Gaussian measurement noise with zero mean and $1 \%$ variance cannot be neglected. This is due to the fact that the vertical stiffnesses of the tires have very little effect on the displacement of the suspensions.

\section{Effects of Polynomial Chaos Approximation}

The accuracy of the estimation depends on the polynomial chaos approximations that are used, i.e., on how many terms are used in the polynomial chaos expansions and how many collocation points are used. Increasing the number of polynomial chaos terms or the number of collocation points also increases the time it takes to compute the results. Table 2 shows the influence of the number of terms used in the polynomial chaos expansions and the number of collocation points. 
The three columns correspond to three number of terms used for the polynomial chaos expansions (10,15 and 21). For each column, four estimation results are displayed. The first one corresponds to a number of collocation points equal to the number of polynomial chaos terms; the second one corresponds to a number of collocation points roughly equal to $150 \%$ of the number of polynomial chaos terms; the third one corresponds to a number of collocation points roughly equal to double the number of polynomial chaos terms, and the fourth one corresponds to a number of collocation points roughly equal to triple the number of polynomial chaos terms.

Table 2. Effect of the Polynomial Chaos Approximations

\begin{tabular}{|c|c|c|c|}
\hline $\begin{array}{c}\text { Number of } \\
\text { Collocation } \\
\text { Points }\end{array}$ & $\mathbf{1 0}$ terms & $\mathbf{1 5}$ terms & $\mathbf{2 1}$ terms \\
\hline 10 & $0.2398,-0.3875$ & & \\
\hline 15 & $0.2456,-0.3968$ & $0.2107,-0.3459$ & \\
\hline 21 & $0.2494,-0.3909$ & $0.2514,-0.3853$ & $0.4878,-0.3915$ \\
\hline 30 & $0.2492,-0.3918$ & $0.2460,-0.3783$ & $0.2496,-0.3773$ \\
\hline 40 & & & $0.2516,-0.3770$ \\
\hline 45 & & $0.2498,-0.3837$ & \\
\hline 60 & & & $0.2550,-0.3844$ \\
\hline $\begin{array}{c}\text { Actual } \\
\text { Values }\end{array}$ & $\mathbf{0 . 2 3 2 6 , - \mathbf { - 0 . 3 8 7 5 }}$ & $\mathbf{0 . 2 3 2 6 , - \mathbf { - . 3 8 7 5 }}$ & $\mathbf{0 . 2 3 2 6 , - \mathbf { - 0 . 3 8 7 5 }}$ \\
\hline
\end{tabular}

It can be noticed that increasing the number of collocation points for a fixed number of terms in the polynomial chaos expansions usually yields better estimations. However, for this particular example, it is not the case with 10 polynomial chaos terms, which happens to yield very good results with only 10 collocation points. This is due to chance in that particular case: the noise level clearly affects the results, and the effect of noise level and polynomial chaos approximations happen to cancel each other out. However, when we changed the random sequence in the noise, we always observed that the combination of 10 terms and 10 collocation points resulted in better estimations than the combinations 15 terms / 15 collocation points and 21 terms / 21 collocation points. When using the minimum number of collocation points required to perform the estimation, i.e., a number of collocation points equal to the number of terms, increasing the number of terms therefore results in poorer estimations. This makes sense since solving a system with more unknowns is more complicated, and adding extra information into a least squares algorithm becomes more valuable as the system becomes more complex. When using a higher ratio between the number of collocation points and the number of terms, adding terms does not result in better estimations since the addition of extra precision eventually affects the results less than the effect of the measurement noise. Therefore, increasing the number of terms in the polynomial chaos expansions does not result in better estimations.

As a conclusion, working with a great number of terms in the polynomial chaos expressions does not seem necessary if we assume that there is noise associated to the measurements. It would come at a great computational cost since working with a high ratio between the number of collocation points and the number of terms becomes crucial, and the precision gained by adding more terms in the polynomial chaos expansions would be small compared with the effect of the noise in the measurements. Let's note that the system used in this study is very sensitive to noise since the vertical stiffnesses of the tires have very little effect on the displacement of the suspensions.

\section{Effects of Smoothness of the Road Profile}

Three different types of road profiles are shown in Figure 8: a smooth bump, a non-smooth bump, and a rectangular bump.
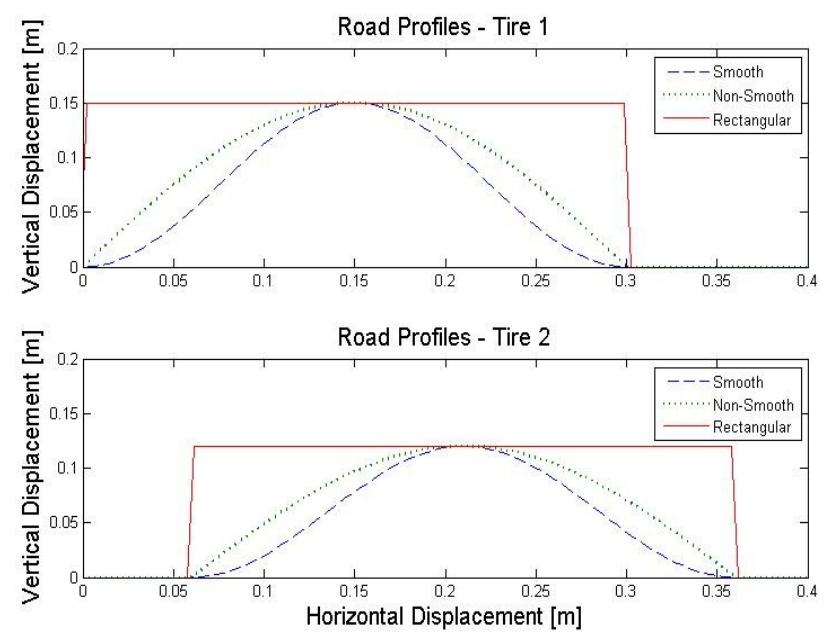

Figure 8. Different Shapes of Road Profiles

The estimations are computed for different sampling rates for those three different road profiles. They are shown in Table 3 , for a speed of $8 \mathrm{~km} / \mathrm{h}(5 \mathrm{mph})$. It is assumed that the observations contain a Gaussian measurement noise with zero mean and $1 \%$ variance for the first three rows of Table 3 . The fourth row shows the effect of having a measurement noise with $0.1 \%$ variance instead of $1 \%$ variance. 
Table 3. Influence of the Smoothness of the Road Profile (15 terms, 30 collocation points)

\begin{tabular}{|c|c|c|c|}
\hline $\begin{array}{c}\text { Sampling } \\
\text { Period }\end{array}$ & $\begin{array}{c}\text { Smooth Road } \\
\text { Profile }\end{array}$ & $\begin{array}{c}\text { Non Smooth } \\
\text { Road Profile }\end{array}$ & $\begin{array}{c}\text { Rectangular } \\
\text { Road Profile }\end{array}$ \\
\hline $0.3 \mathrm{~s}$ & $0.4814,-0.2787$ & $0.5794,-0.5479$ & $0.1887,-0.6274$ \\
\hline $0.1 \mathrm{~s}$ & $0.3766,-0.2650$ & $0.5261,-0.4517$ & $0.3862,-0.7379$ \\
\hline $0.03 \mathrm{~s}$ & $0.2600,-0.3220$ & $0.1412,-0.4711$ & $0.3008,-0.7047$ \\
\hline $\begin{array}{c}0.3 \mathrm{~s} \\
(0.1 \% \text { noise })\end{array}$ & $0.3563,-0.2511$ & $0.5350,-0.3906$ & $0.3990,-0.7405$ \\
\hline $\begin{array}{c}\text { Actual } \\
\text { Values }\end{array}$ & $\mathbf{0 . 2 3 2 6 , - \mathbf { 0 . 3 8 7 5 }}$ & $\mathbf{0 . 2 3 2 6 , - \mathbf { 0 . 3 8 7 5 }}$ & $\mathbf{0 . 2 3 2 6}, \mathbf{- 0 . 3 8 7 5}$ \\
\hline
\end{tabular}

The three road profiles shown in Figure 8 consist of bumps that are much smaller than the one shown in Figure 5. Therefore, estimating the vertical stiffnesses of the tires becomes a tougher task. Table 3 shows that increasing the sampling period of the measurements results in better estimates. The effect of having a measurement noise divided by 10 on the quality of the estimation is approximately the same than the effect of increasing the sampling period by 3 for this particular example. What is consistent is that the shape of the input clearly affects the results of the estimation. The smooth road profile yields the best results: the estimation is quite accurate for a sampling period of $0.03 \mathrm{~s}$. The non-smooth profile (with a noncontinuous derivative) does not yield very accurate results. The rectangular (non-continuous) road profile yields poor results even for a sampling period of $0.03 \mathrm{~s}$.

\section{BAYESIAN APPROACH VERSUS EXTENDED KALMAN FILTER (EKF) APPROACH}

In a pending article by the authors [1], we consider the problem of finding an updated polynomial chaos representation of the state which accounts for all the observations, given the polynomial chaos representation of the current state $y$. Since a direct application of Bayes' formula seems difficult, a simplified (but sub-optimal) variant based on the Extended Kalman Filter (EKF) is considered. While the Kalman filter [3, $4,5,8]$ assumes that the model is linear, and the model state at previous time is normally distributed, the Extended Kalman Filter (EKF) allows for nonlinear models and observations by assuming that the error propagation is linear. In the EKF approach the nonlinear observation operators are linearized.

The EKF estimations come in the form of Probability Density Functions, whereas the Bayesian approach estimations only consist of deterministic values. However, the EKF approach is sub-optimal for non-Gaussian uncertainties, whereas the Bayesian approach is not tailored to any specific distribution. Future work will include a rigorous comparison of the two approaches on several tests cases.

\section{CONCLUSIONS}

This paper applies the polynomial chaos theory to the problem of parameter estimation, using direct stochastic collocation. The maximum likelihood estimates are obtained by minimizing a cost function derived from the Bayesian theorem. Parameter estimation is performed on a four degree of freedom roll plane model of a vehicle, where uncertainties on the values of the vertical stiffnesses of the tires are assumed to have a Beta $(1,1)$ distribution. The proposed Bayesian approach resulted in a good estimation of the vertical stiffnesses of the tires for a large road input. However, it is shown that measurement noise can have a negative impact on the quality of the estimations, especially for short road inputs. The smoothness of the road profile also affects the results.

The accuracy of the estimations has been shown to be sensitive to the number of terms used in the polynomial chaos expressions and to the number of collocation points. However, it is usually not necessary to use a large number of terms in the polynomial expressions since the addition of extra precision eventually affects the results less than the effect of the measurement noise. Increasing the number of terms in the polynomial chaos expansions is actually a bad idea when using a small ratio between the number of collocation points and the number: it yields poorer estimations.

The proposed estimation procedure can work with noisy measurements. The problem with the system used in this study is that the vertical stiffnesses of the tires have very little effect on the displacement of the suspensions. This explains why the results of the estimations are quite sensitive to a small level of noise in the measurements. Estimating the vertical stiffnesses of the tires based on the behavior of the suspensions is not a very realistic task since the other parameters of the system would have to be perfectly known due to their greater effect on the displacements of the suspensions. Future work will include estimation of different parameters.

The proposed method has several advantages. Simulations using Polynomial Chaos methods are much faster than Monte Carlo simulations. Another advantage of this method is that it is optimal; it can treat non-Gaussian uncertainties. However, a few issues exist with this approach. The cost function can have multiple local minima, which can affect the estimates when dealing with large measurement noise. These issues will be investigated in the future. The proposed estimation procedure may benefit from regularization techniques. Future work will 
also include a rigorous comparison of the Bayesian approach with a variant based on the Extended Kalman Filter (EKF).

\section{ACKNOWLEDGMENTS}

This research was supported in part by NASA Langley through the Virginia Institute for Performance Engineering and Research award.

\section{REFERENCES}

[1] Blanchard, E., Sandu, A., and Sandu, C. - "Parameter Estimation Method Using an Extended Kalman Filter", Proceedings of the Joint North America, Asia-Pacific ISTVS Conference and Annual Meeting of Japanese Society for Terramechanics, June 23-26, 2007, Fairbanks, Alaska.

[2] Cohn, S. E. "An Introduction to Estimation Theory", $J$. Meteor. Soc. Japan 75 (B) (1997), 257-288.

[3] Evensen, G. "Using the Extended Kalman Filter with a Multi-layer Quasi-geostrophic Ocean Model”, J. Geophys. Res. 97 (C11) (1992), 17905-17924.

[4] Evensen, G. "Open Boundary Conditions for the Extended Kalman Filter with a Quasi-geostrophic Mode", $J$. Geophys. Res. 98 (C19) (1993), 16529-16546.

[5] Fisher, M. Assimilation Techniques(5): Approximate Kalman Filters and singular vectors (2002)

[6] Halton, J. H., Smith, G. B. "Radical-inverse quasi-random point sequence". Communications of the ACM, 7(12):701-702, Dec. 1964.

[7] Hammersley, J. M. "Monte Carlo Methods for Solving Multivariables Problems", Ann. New York Acad. Sci., 86:844874, 1960.
[8] Kalman, R. E. "A New Approach to Linear Filtering and Prediction Problems", Transaction of the ASME- Journal of Basic Engineering (1960), 35-45.

[9] Sandu, A., Sandu, C., and Ahmadian, M. - Modeling Multibody Dynamic Systems With Uncertainties. Part I: Theoretical and Computational Aspects, Multibody System Dynamics, Publisher: Springer Netherlands, ISSN: 13845640 (Paper) 1573-272X (Online), DOI 10.1007/s11044006-9007-5, pp. 1-23 (23), June 29, 2006.

[10] Sandu, C., Sandu, A., and Ahmadian, M. - Modeling Multibody Dynamic Systems With Uncertainties. Part II: Numerical Applications, Multibody System Dynamics, Publisher: Springer Netherlands, ISSN: 1384-5640 (Paper) 1573-272X (Online), DOI: 10.1007/s11044-006-9008-4, Vol. 15, No. 3, pp. 241 - 262 (22), April 2006.

[11] Simon, D. E. "An Investigation of the Effectiveness of Skyhook Suspensions for Controlling Roll Dynamics of Sport Utility Vehicles Using Magneto-Rheological Dampers", Ph.D. Thesis, Virginia Tech, Blacksburg, VA, 24061, 2001.

[12] Sohns, B., Allison, J., Fathy, H. K., Stein, J. L. "Efficient Parameterization of Large-Scale Dynamic Models Through the Use of Activity Analysis", Proceedings of the ASME IMECE 2006, IMECE2006, Nov 5-10, 2006, Chicago, Illinois.

[13] Zhang, D., Lu., Z. "An efficient, high-order perturbation approach for flow in random porous media via Karhunen-Loeve and polynomial expansions", J Comp. Phys. 54:265-291 (2006) 